\title{
TREATMENT OF BOUNDARY CONDITIONS IN THE ANALYSIS OF PBG STRUCTURES
}

B2: Finite Element/ C8: Photonic

K.S.Thomas ${ }^{1}$, M. Molinari ${ }^{2}$, S.J. Cox ${ }^{2}$

${ }^{1}$ School of Electronics and Computer Science

${ }^{2}$ School of Engineering Sciences

University of Southampton

Highfield, Southampton

SO17 1BJ, UK

\{kst@ecs.soton.ac.uk, m.molinari@ soton.ac.uk, sjc@soton.ac.uk\}

\section{ABSTRACT}

Floquet theory is used in the analysis of periodic structures such as photonic crystals. We argue in this paper, that we can use the first form of the Floquet transform to treat the boundary conditions. The advantage is that we can use standard (nonperiodic) software for the analysis by the finite-element method. We give an example of this technique to the electromagnetic Kronig-Penny model in three space dimensions, where the use of vector elements is essential.

\section{INTRODUCTION}

Photonic Band Gap (PBG) structures are periodic dielectric crystals that exhibit a photonic band gap. Photons in the frequency range of the band gap are completely excluded. PBGs offer great potential in the development of highly efficient narrow band (tuneable) lasers. Because of high costs in manufacture, theoretical models to predict the behaviour are paramount. The equations defining the properties of the photonic crystals are defined over the whole of three dimensional space and we seek solutions that are periodic. Fundamental is the Floquet transform and in this section we follow the treatment of [4] who defines the Floquet transform $U f$ of a function $f(r)$ by

$$
U f(r, k)=\sum_{n \in \mathbb{Z}^{3}} f(r-n) e^{i k . n}
$$

where the vector $k$ is called the quasimomentum. This is similar to the Fourier transform but is distinct in the sense that the transform depends on both the position and the quasimomentum. A fundamental property of this form of the Floquet transform is commutability with linear differential operators.

Thus the transformation of Maxwell's equations will lead to identical equations defined of the unit cube. The boundary values will arise from the Floquet condition

$$
(U f)(r+m, k)=e^{i k . m}(U f)(r, k), m \in \mathbb{Z}^{3}
$$

The Floquet transform is periodic with respect to the quasimomentum $k$. Thus to determine $U f(r, k)$ we only need values for $k$ belonging to the dual lattice $2 \pi \mathbb{Z}^{3}$. This region defines the Brillouin zone.
The most common method of imposing periodicity is to set $E=e^{i k . r} E_{0}$ and leads to equations in which the gradient operator $\nabla$ is replaced by $\nabla+i k$. The boundary condition is periodicity, imposed as an essential boundary condition. Theoretically, there are some difficulties that are noted in [2] who add phase transitions the Nedelec edge elements. But we argue that our formulation is just as effective, leading to standard equations and enabling existing research to be exploited. All that is needed is a study of boundary conditions that arise from (2).

\section{FINITE ELEMENT ANALYSIS}

We consider the differential equations that arise from the elimination of the magnetic field $H$ from Maxwell's equation. The vector valued function $E$ is the Floquet transform of the electric field.

$$
\begin{aligned}
& \nabla \times \frac{1}{\mu} \nabla \times E=\omega^{2} \varepsilon E \\
& \nabla \cdot(\varepsilon E)=0
\end{aligned}
$$

The essential boundary condition in our system is that $E$ satisfies (2). A natural boundary condition is that $\nabla \times E$ also satisfies (2) and this can be verified when we derive the weak form of the equations by integration by parts.

$$
\int_{\Omega} \frac{1}{\mu} \nabla \times E \cdot \nabla \times \bar{F} d V=\omega^{2} \int_{\Omega} \varepsilon E \cdot \bar{F} d V
$$

where $\Omega$ is the unit cube.

Our domain is meshed using the package NETGEN [7] that can be made to produce periodic meshes. These are essential if we are to avoid issues of nonconformance. We also ensure that the mesh aligns with the different material regions. Thus the permittivity $\varepsilon$ is piecewise constant in each tetrahedron.

In each tetrahedron, we use lowest order Whitney element described in [6],[8]. Given a set of basis functions $\left\{\phi_{i}\right\}$ construction of the stiffness matrix $A$ and mass matrix $B$ is straightforward and given in [3]. These two matrices are defined by

$$
\begin{aligned}
& A=\left\{\int_{\Omega} \frac{1}{\mu} \nabla \times \phi_{i} \cdot \nabla \times \phi_{j} d V\right\} \\
& B=\left\{\int_{\Omega} \varepsilon \phi_{i} \cdot \phi_{j} d V\right\}
\end{aligned}
$$

Both these matrices are real and symmetric and do not depend on the quasimomentum.

A constraint matrix $C$ is introduced to impose the Floquet condition. It is important that this matrix is of full rank. To indicate the construction, we consider a degree of freedom on the face $x=0, u_{p}$ and its periodic image on the face $x=1, u_{q}$. The Floquet condition is then

$$
e^{i k_{1}} u_{p}=u_{q}
$$


We can derive equations in this way for all periodic pairings in the system. However, a naïve approach will not ensure full rank of the matrix $C$. Full rank is ensured by enforcing the rule that an individual degree of freedom appears on the right hand side of only one constraint equation.

\section{LINEAR ALGEBRA}

Having assembled the stiffness, mass and constraint matrices, the computational problem is the solution of the generalised eigenvalue problem

$$
\left[\begin{array}{cc}
A & C^{*} \\
C & O
\end{array}\right]\left[\begin{array}{l}
u \\
v
\end{array}\right]=\omega^{2}\left[\begin{array}{ll}
B & O \\
O & O
\end{array}\right]\left[\begin{array}{l}
u \\
v
\end{array}\right]
$$

In this partitioned matrix, only the constraint matrix depends on the quasimomentum. The number of rows in $C$ is significantly smaller than the size of $A$.

Subspace iteration is an effective method of finding the spectrum. When a shift $\sigma$ is introduced the iteration reduces to the solution of linear equations with coefficient matrix

$$
\left[\begin{array}{cc}
A-\sigma B & C^{*} \\
C & O
\end{array}\right]
$$

The shift is chosen to amplify the lowest nonzero eigenvalues and to ensure that the matrix $A-\sigma B$ is non-singular. This last property and the full rank of the matrix $C$, ensures that the matrix is non-singular.

\section{EXAMPLE}

The Electromagnetic Kronig-Penney model, considered in [5] is a repeated pattern of two layers of materials of differing dielectric constant(Figure 1). This was taken as our test case. Because of symmetry, the quasimomentum was restricted to one dimension. The results are given in Figure 2

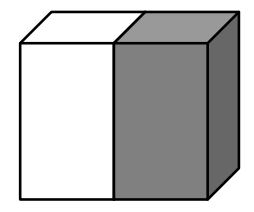

Figure 1. A unit cell in the EKP model. The dielectric constant $\varepsilon$ is unity in the white region 9 in the grey region.

\section{CONCLUSION}

This treatment of the Floquet boundary conditions has several advantages. The main advantage is that we can use the software for nonperiodic problems. The construction of the stiffness and mass matrices is identical. The theoretical analysis of mixed problems [1] is applicable to the problem area of computing PBGs in photonic crystal structures. Thus higher order vector elements can be used to some advantage.
The disadvantage is a small increase in the sizes of matrices involved in the linear algebra; but we have observed that this is not significant.

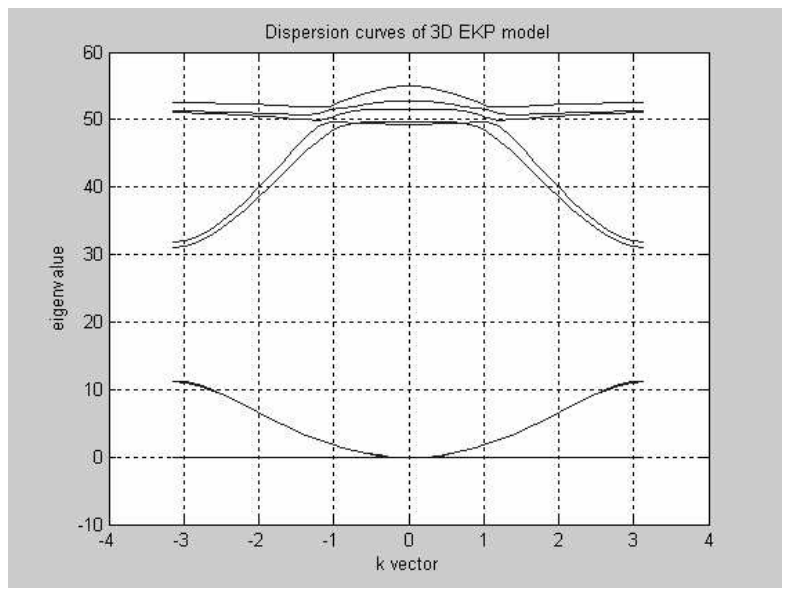

Figure 2. Computed dispersion curves: the discretisation had 863 degrees of freedom and there were 144 constraints.

\section{REFERENCES}

1. Boffi, D., et al., Computational models of electromagnetic resonators: Analysis of edge element approximation. Siam Journal on Numerical Analysis, 1999. 36(4): p. 12641290.

2. Dobson, D.C. and J.E. Pasciak, Analysis of an algorithm for computing electromagnetic Bloch modes using Nedelec spaces. Computational methods in applied mathematics, 2001. 1(2): p. 138-153.

3. Jin, J., The finite element method in electromagnetics. 1993: Wiley.

4. Kuchment, P., The Mathematics of Photonic Crystals, in Mathematical Modeling in Optical Science, G. Bao, L. Cowsar, and W. Masters, Editors. 2001, SIAM: Philadelphia.

5. Mias, C., J.P. Webb, and R.L. Ferrari, Finite element modelling of electromagnetic waves in doubly and triply periodic structures. Iee Proceedings-Optoelectronics, 1999. 146(2): p. 111-118.

6. Nedelec, J.C., A New Family of Mixed Finite Elements in R3. Numer. Math, 1980. 50: p. 57-81.

7. Schöberl, J., NETGEN automatic mesh generator. 2003. http://www.hpfem.jku.at/netgen/

8. Silvester, P.P. and R.L. Ferrari, Finite Elements for Electrical Engineers. Third ed. 1996: Cambridge University Press. 